\title{
A Data-Driven Fatigue Prediction using Recurrent Neural Networks.
}

\author{
Arsalan Lambay \\ School of Engineering \\ Cardiff University \\ Cardiff, United Kingdom \\ lambaya@cardiff.ac.uk \\ Phillip Morgan \\ School of Psychology \\ Cardiff University \\ Cardiff, United Kingdom \\ morganphil@cardiff.ac.uk
}

\begin{abstract}
Industrial revolution 4.0 has marked the era of advances in interaction among machines and humans and cultivate automation. However, manufacturing industries still have tasks which are labor intensive for humans with lots of repetitive actions. These actions along with other factors can cause the worker to be fatigued or exhausted. These in the long term can develop into work-related musculoskeletal disorders (WMSD). Nevertheless, comprehending fatigue in a quantifiable and objective manner is yet an open problem due to the heterogeneity of subjects involved for data collection.
\end{abstract}

In this study a benchmarking dataset comprising of physical fatigue attributes. They are used to perform fatigue prediction for manual material handling task. It includes data collected from Inertial Measurement unit (IMU) and Heart Rate (HR) sensor which is then pre-processed to extract to be used to run the model. The data serves as an input to a time-series prediction model called as Recurrent Neural Network (RNN).

Keywords-Manufacturing, Fatigue Prediction, WMSD, Recurrent Neural Networks

\section{INTRODUCTION}

The manufacturing industry has witnessed the rise in advance automation for over a decade. However, labour demanding task are still dominant in manufacturing industry. These tasks involve physical exertion and mental straining, causing fatigue. It makes it necessary to manage operator's fatigue, else it will hamper the work quality, reduce productivity, and could cause incidents and accidents. Nina Vøllestads defined fatigue as "any exerciseinduced reduction in the maximal capacity to generate force or power output" [1]. There are several factors that govern fatigue in an individual such as social-economic, psychological, working environment, etc. Fatigue depends on an individual's perspective, i.e., it is sensation based, multi-dimensional construct. It reduces an operator's ability to concentrate, hindering one's performance. In 1914, Sir Thomas Oliver first coined the term occupational fatigue which involved the new science of understanting industrial pysiology [2]. In essence to operator's safety and health, makes it conspicuous to study fatigue for its short and long term effects. Further-more it can't be separated into physical and mental fatigue. Physcial fatigue is defined as the diminishing ability of an operator to perform physcial task due to excessive phycial exertion. On the contrary, mental fatigue is due the excessive cognitive activities [3].

\author{
Ying Liu \\ School of Engineering \\ Cardiff University \\ Cardiff, United Kingdom \\ liuy81@ cardiff.ac.uk \\ Ze Ji \\ School of Engineering \\ Cardiff University \\ Cardiff, United Kingdom \\ jiz1@cardiff.ac.uk
}

In this report focussing only on physcial fatigue as this seems to be the most prevlent in manufacturing environments. Short- term effects include constriction of motor control, increased malise and reduced physcial capacity and these and other effects, can lead to accidents [4]. Over the time short-term effects can develop into more adverse effect such as Chronic Fatigue Syndrome (CFS) and Work-related Musculoskeletal Disorders (WMSD) [5] [6]. The prolonged effects of WMSD are related to significant health and economic depresiation. These effects are due to the nerve, ligament, muscle, joints and spinal injuries in the long term work.

Under I4.0 workplace automation has increased but interestingly the workload and the fatigue induced has also increased in the workers [7]. Many studies show that tasks with labor instensive physical exertion cause WMSD. Reports such as 'Safety and Health Assessemnt and Research for Prevention'(SHARP) and 'Occupational Saftey and Health Administration' (OSHA) revels that 50\% of the workforce has WMSD and $34 \%$ of lost work days due to it, respectively. Other industries such as construction and manual material handling (distribution) have also reported high fatigue rate amoung its workers [8].

The European Union, Sweden, Canada and Japan have also reported for fatigue amoung the workers after the shift works [5] [9] [10] [11]. Working hours, extensive work load, unevenly organised shifts (especially night shifts), insufficient break time for recovery from fatigue (i.e., rest), and craptastic working environments are among other factors that can cause fatigue [8]. The cost of fatigue is not only beared by the worker but the organization as well which is in two ways economic cost and lost working days. Ricci et. al. (2007) [12] faound that fatigue cost $83.9 \%$ in the lost productivity time which amounts to $\$ 136$ billion anually. It reinforces a cost of $\$ 20$ billion in compensation to the employer related to WMSD alone. The report by OSHA also outlines that by and large one out of three dollar is spent directly on WMSD [13]. Based on the issues identified of fatigue on individiuals performance and health the current studies sets out to investigate prediction of fatigue to mitigate its effect. It is noteworthy to predict fatigue inducing in the worker for the aforementioned reason as it cost substantially to both worker and organization. 


\section{II. .REALTED STUDIES}

A feeling of fatigue in an individual is sensation based and completely varies for every individual. This makes it challenging to quantify the level of fatigue induced. However, fatigued has been analyzed in different ways. They are subjective based, objective based, and data-driven type.

\section{A. Subjecive Based Analysis}

Questionnaire-based analyses are subjective based, often involving self-report rating of = individual discomfort. These tend to focus on the workers feelings of fatigue and consequences. Wiktorin et. al. (993) [14] developed a method consisting of questionnaires and interviews to try and understand the level of fatigue in workers. Among these (the widely adapted) is used is 'Rated Perceived Exertion (RPE)' scale, a technique which is used to assess the muscle fatigue, also called the 'Borg Test' [15]. Borg scales ranges from 6 to 20 with every increase in the in the scale reflecting maximum exertion. It depends on the self-perceived rating of an individual which differs with every individual. Furthermore, the Swedish Occupational Fatigue Inventory (SOFI) follows a similar principle of RPE and accounts five main aspects of fatigue: lack of motivation, physical discomfort, physical exertion, sleepiness, and energy/effort [16]. A relatively new self-administrated questionnaire system to differentiate between motor and cognitive fatigue is known as 'Fatigue Scale for Motor and Cognition' (FSMC) [17]. Aforementioned methods do take in account the sensation but lack immediate results and limit in intermittent recording techniques which lack the usefulness in real time.

\section{B. Objective Based Analysis}

Objective based fatigue analyses measure involves the analysis of body parts which exert the force required during the task such as using ergonomic tool posture-based analysis. Three posture-based analysis tools were developed in 1999: 'Posturegram', Ovako Working Posture Analyzing System (OWAS)' and 'Quick Exposure Check' [18]. All three techniques work on simlar principle with predefined rules to rank working posture whilst, observing risk level. While QEC also takes in account the amount of exposure to physical exertion. However there are some tools such as 'Rapid Upper Limb Assessment' (RULA) [19], 'HandArm-Movemnet Analysis' (HAMA) and 'PLIBEL which focused on posture and musculoskeletal stress of hand movemnet co-ordination [20]. These methods were limitied to posture analysis and not specfic in determining the fatigue state. Alternatively, Williams and Rogers designed'Muscle Fatigue Analysis' (MFA), using a similar principle but focousing on fatigue analysis [21]. Body party were segregated with the help of working position, the span of effort and time required for the task in to four levels. Depending on these assessemnt a prioprity score would be calculated and the task with high prioprity score would be redisigned to reduce the fatigue [20]. The limitation was that these techniques were lab-based.

\section{Data-Driven Analysis}

A data-driven analysis is a technique which collects data from sensors to predict fatigue and is one of the widely adopted techniques to measure fatigue through machine learning. There are two ways in which data-driven analysis can be divided into: First are the invasive data collection techniques and secondly the non-invasive data collection. A relatively new acclimated technique which has been adopted is the wearable sensors.

1) Invasive Approaches: these employs biomedical sensors which measure the metabolic changes. These changes are detected by the sensor and machine learning algorithm used to classify a fatigue state in an individual. The most popularly used is Electromygraphy (EMG) senors. EMG captures the electrical activity (neuronal firninh) generated from the central nervous system under the skin (motor units). The reults are displayed in the form of power spectrum and signal amplitude. The neurons supplied are directly proportional to the amplitude measured. A study by Isa et. al. in 2014 [22] was carried out to study fatigue of differnt muscle in a manual material handling task. Similarly, an amalgamation of EMG and 3D accelerometer was used to study fatigue for upper arm by employing a Principal Component Analysis (PCA) classification technique to predict fatigue [23]. Although, it was restricted to nerve stimulation and not considering changes in muscle fibre. Kider et al. [24] used Electrocardiography (EKG) and Galvanic Skin response (GSR) to measure fatige. EKG captured heart movements elecartical volatge whereas GSR captured the electrical conductance from the skin. These methods provided noteable results of, however they cannot be used in real world application, restricting to lab based. Invasive approach does privide a semaless approach and interweave with the working process causing discomfort.

2) Non-Invasive approach: These do not involve attaching sensory devices to the body and thus are noninvasive. Different approaches such as motion capture, force sensors and behavioral analysis have been used to measure fatigue. Motion capture are sometimes combined with a different invasive sensors coupled machine learning algorithm to measure fatigue [25]. This technique are popular in driver fatigue assement but are expensive. Altenatively, using force sensors to predict fatigue by Pradip et al [26], and employed CUSUM algorithm. Force sensors used in plates to study ground and foot reaction forces were used. The drawback was high number of plates were required as a person walks in continous manner and this made it complex for modelling challenging to integrate consecutive plates. However, there are some other techniques, using behavioral charasteristic to predict fatigue such as keystroke dynamic, gait, etc. [27]. These techniques use behavioural changes in an individual to measure fatigue.

3) Wearable sensor approach: these are less invasive and having less discomfort. An popular example of wearbale is Inertial Measurement Unit (IMU). It assess gait parameter of an individual to predict the onset of fatigue.

4) Machine Learning: is a strand of artificial intelligence(AI) which uses data as input to extract knowledge in the form of patterns to learn from it and improve it over time to predict fatigue. In research papers, the most frequently used ML techniques for prediction are Support Vector Machine (SVM), Linear Discriminant 
Analysis (LDA), k-nearest neighbors (KNN), Naive Bayes (NB) and Penalisad Regression Models [28] [26] [27] [5]. As fatigue is a multidimensional construct there were attempts to mimic human brain operation by using Neural networks. It is mostly concentrated towards studying driver fatigued. Although, recurrent neural networks have been employed to measure fatigue but used for motion capture. It has been derived from feed forward network and is used for producing predictive results for sequential data.

\section{METHEDOLOGY}

This section gives a brief about the method used for predicting the fatigue.

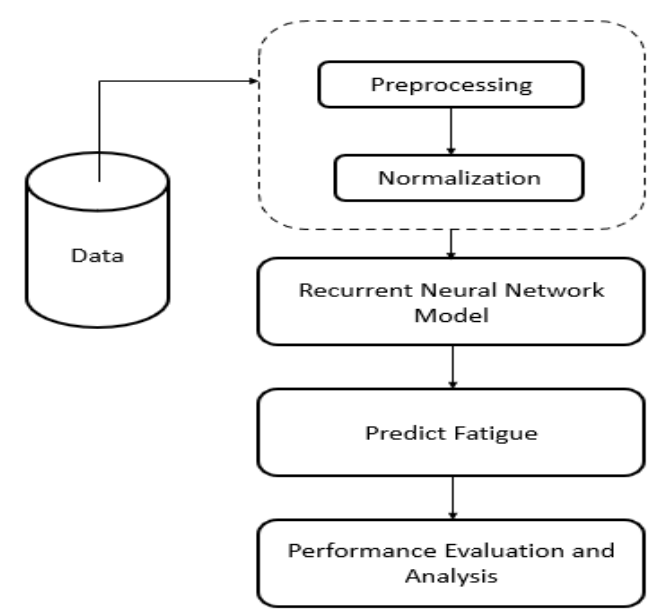

Figure 1: Fatigue Prediction Model

\section{A. Data}

The data for fatigue prediction was obtained from benchmarking data collected from different sensors. The dataset contained data about the demographic variables such as age gender, RPE rated fatigue and data inputs from mainly two sensors, 'Inertial Measurement Unit' and Heart Rate Monitor for which the data was present in tabular format [28].

\section{B. Preprocessing of Data}

In any machine learning application data preprocessing plays a pivotal role for improving the efficiency of the model. This helps in choosing the best models by sensing a deeper understanding of the data. Once the model is chosen, the data can be pre-processed to optimize the learning or the best way in which the model can understand. A benchmarking dataset was used to train an RNN-LSTM network which comprised of floats and integers. In order to ameliorate the accuracy of machine learning model, normalization was applied to all data in the features. The data of each feature that is used by the LSTM model is normalized. To build the recurrent neural network, we use normalization technique i.e., subtracting the minimum value of the data set and then dividing by the range of the data set. Furthermore, for scaling the range of the data the 'MinMaxScaler' class lives within the preprocessing module.

\section{Recurrent Neural Network Model}

Recurrent neural network is providing a solution to a time series prediction problem. RNN is a type of neural network (NN) where nodes are connected in such a way to form a sequence of data [29]. The Keras neural network Application Programming Interface (API) is used to construct and train the LSTM model. The API is a Pythonbased open-source deep learning framework that leverages TensorFlow as a backend. Keras's quick learning curve, combined with its ease of implementing deep learning models, make it an excellent tool for this project. Moreover, TensorFlow, also open source is a machine learning framework for numerical computations.

$\mathrm{RNN}$ is a NN used for predicting time dependent solution, uses internal memory remembering from past observation to feed the network. The usage of RNN with the preprocessing methods outlined validates the usage of NN for both supervised and unsupervised learning discussed earlier in this section. An RNN makes a prediction by combining the input data and prior outputs, which is exactly what is needed to predict fatigue based on previous task motions. However, the RNN has a limitation of suffering from short term memory. The network mainly encounters two problems: vanishing gradient or exploding gradient. This means it struggles to carry previous observation. When training the RNN, back propagation is used to calculate the gradient through the layers. These gradients to update the weight of the networks suddenly becomes too large (exploding gradient) or too small (vanishing gradient). Long Short-Term Memory and Gated Recurrent Unit are two popular RNN based solution for the gradient problems which work well with the time series prediction. They both use gates in order to manage the memory contents of NN. An input gate, a forget gate, and an output gate are all used in the LSTM. The internal state will be managed by the input and forget gate, which will decide what to save and when to erase. [30]. The GRU uses gates in the same way that the LSTM does, except it only has two instead of three. There is an update gate that regulates the flow of data and specifies how much of the previous observation should be passed on to the future, as well as a reset gate that indicates how much of the previous data should be forgotten. GRU is nothing more than a simplified form of LSTM. As it has been demonstrated that RNNs can anticipate human motion, it is possible to forecast fatigue for a specific material handling operation by integrating the ability to anticipate sensory input for fatigue analysis. As a result, the machine learning methodology presented will be based on RNN techniques. The LSTM, 3-Layer LSTM, and 4-Layer LSTM models will be employed.

\section{Performance Evaluation}

The evaluation of the performance of the machine learning models, the performance measures used are accuracy, the percentage of correct classifications made by a given model. Practitioners can use this metric to see if a model is capable of predicting both fatigued and non-fatigued states equally well. The mathematical formula for accuracy is stated below: 


$$
\text { Accuracy }=\frac{T P+T N}{T P+T N+F P+F N}
$$

where TP, TN, FP, FN denote the number of true positives, true negatives, false positives, and false negatives, respectively.

\section{EXPERIMENTAL ANALYSIS}

Long Short-Term Memory (LSTM) was the best architecture that worked well for us. The LSTM layer directly after the input layer, followed by four dense layers. The LSTM layer output size was 20 . The dense layers had 32 neurons in each of the layer. Rectified Linear Units (ReLU) with a linear identity activation function for input values above zero and all zero output for input values below or equal to zero were used in the dense layer neurons. There are several objectives that have been met by completing classification and regression tasks. For classification tasks, one neuron with SoftMax activation function was used and one with ReLU neuron was used. Mean Squared Error was chosen as the error metric and loss function, while the binary cross-entropy was chosen for the classification job. A maximum of 100 epochs for training were used. The implementation was done in Python (v3.8.5) with the TensorFlow package (v2.3.0) [31]. Every run was repeated numerous times to guarantee that the model's robustness and the entire training and evaluation process were not accidental. Furthermore, the network operations' random seed was not set to a fixed value but was chosen at random each time. Leave-One-Out-Cross (LOOCV) validation was used to assess all networks. The purpose of the generalized analysis was to train the neural networks on patterns that were common to all participants, therefore all recordings from one participant were left out of training for each test and validation set. To obtain answers for both options, the analysis was separated into a distinctive and a generalized analysis.

\section{A. Input}

The representation every fatigue was by RPE scale in our dataset as a vector of the size $1 \times 23$, where 23 is the number of features which were include in our prediction. We created a matrix with all of the Fatigue RPE scale and their features that are considered in the prediction and divided all of the tasks into fixed-size windows. Then we reshape all of the matrix's vectors to create a NumPy array. It is now a shape vector ( $\mathrm{W}-1-\mathrm{f})$, where $\mathrm{W}$ is the number of windows, 1 is the length of $A$ window, and $f$ is the number of features. The dataset was then divided into train and test sets, with the training set accounting for $70 \%$ of the dataset and the test set accounting for $30 \%$. As it has the highest significance, we train the LSTM Model to determine the tiredness of the modified by different feature input for each training.

\section{B. Hidden Layers}

We must evaluate how many hidden layers the model will contain, the number of LSTM cells that should be included in each layer, and what the dropout should be when building the LSTM model. However, there is no universally accepted rule for determining the number of hidden layers or cells within each layer. The successful models are: one with four layers, and the other with three layers and. The number of cells and layers depends on the application for which the LSTM model will be used, and the cells in each layer should have the same number of cells for finding an optimal structure. A dense layer is a densely connected NN layer in which each cell in the following layer is connected by a dense layer. There are also successful models that use dense layers by first creating a model of number of hidden layers, then adding many dense layers.

There are hyperparameters that must be appropriately setup and modified while developing the LSTM model so that we may achieve an accurate fatigue prediction while testing our model. In this study, we conducted empirical experiments to determine the best hyperparameters for improving accuracy and reducing the danger of overfitting the data.

Dropout is a useful approach for reducing overfitting by selecting cells in a layer at random based on the probability chosen and setting their output to 0 . The ideal degree of dropout was determined using an empirical test, which was then applied to all of the hidden layers. We developed our LSTM model, trained it, and then varied the dropout values so that the difference between consecutive dropout values was constant. The dropout is set to $20 \%$. The optimal value of dropout set was $0.2(20 \%)$ in our case as it has the smallest mean squared error. While doing this test the epoch was set to 100, the LSTM cells in each layer was set to $32,64,128,1$, decay to 0.2 .

An epoch occurs when all of the training data has been sent through the network; thus, one epoch corresponds to one repetition of the entire training data passing through the network. When the training data is propagated through the network, we divide the training data into the batches' sizes of 16. This indicates that the first 16 samples from the training set (0-16) are used to train the network, and then the next 16 samples are used to train the network. The epoch will continue until all samples have been transmitted across the network. The optimal epoch value in our case is 100 as it showed the lowest value of MSE.

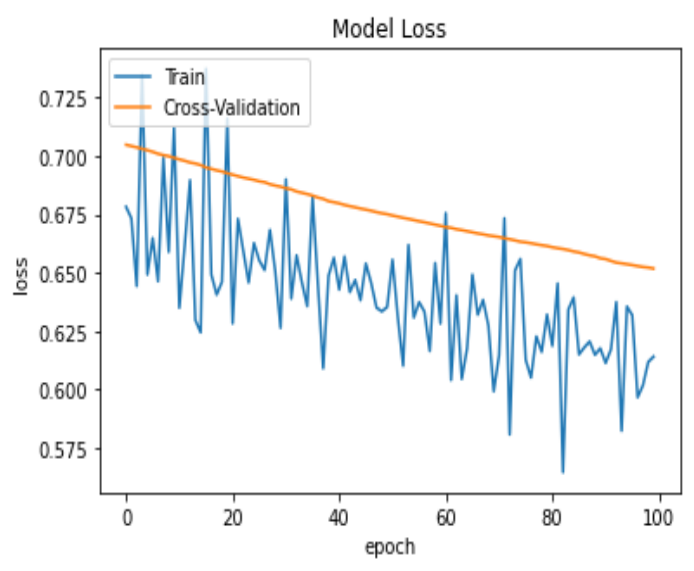

Fig 2 Epoch vs Loss

During training, the loss function quantifies the distance between the LSTM model's output and the desired output 
to speed up learning. The validation data, which is set by the user, is the desired result; we have chosen to make the validation data $50 \%$ of the training data. As the training data output is compared to the validation data after each epoch, this can prevent overfitting by stopping the model during training. We may be overfitting if the training loss is falling, and the validation is growing at the same time. The MSE was chosen as our loss function because it is commonly used for time series forecasting when selecting a loss function. The mathematical formula for the same is given below:

$$
M S E=\frac{\sum_{i=1}^{n}\left(y i-y_{i}^{p}\right)}{n}
$$

Where, $\mathrm{n}=$ samples, $\mathrm{i}=$ boss trapping samples, $\mathrm{y}=$ predicted values

\section{Dense Layers}

A dense layer is a densely connected NN layer, where each cell of a dense layer is connected to another dense layer in the next layer. The successful models using dense layer by building their model of hidden layers followed by multiple dense layers. The LSTM model in our case contains four layers, two hidden layers and one dense layer. The first hidden layer's output is connected to another hidden layer, which is then connected to a dense layer, i.e., hidden layer! hidden layer! dense layer! were! Represent the connection between layers. To avoid the possibility of overfitting, dropouts are utilized after each hidden layer.

\section{RESULT AND DISCUSSION}

The performance results are briefly discussed in this part, as well as their comparison to the proposed model. This section briefly discusses neural network hyper-parameters, test and validation findings, and graphical representations of outcomes for each baseline technique. The results are shown in the form of a table at the end of the section in comparison to the proposed model.

\section{A. Accuracy}

The accuracy of the LSTM neural network is shown in table below. The accuracy of the model for training, validation and test is shown.

TABLE I. Accuracy

\begin{tabular}{|l|c|c|c|}
\hline \multirow{2}{*}{$\begin{array}{c}\text { Table } \\
\text { Head }\end{array}$} & \multicolumn{3}{|c|}{ Accuracy } \\
\cline { 2 - 4 } & Training data & Validation Data & Test Data \\
\hline NN & 0.63 & 0.70 & 0.65 \\
\hline
\end{tabular}

The accuracy for training, validation and testing is $63 \%$, $70 \%$ and $65 \%$ respectively. The accuracy for the test data increases compared to the training data which shows that the model learns better with every epoch according to the nature of RNN, which has the tendency of using output of every layer as input to next layer.

Figure 3 displays, accuracy with every epoch i.e., the training data compared to the cross-validation data set. However, in the graph the validation data observes an increase compared to the training. This shows that after cross-validation the accuracy of predicting fatigue increases.

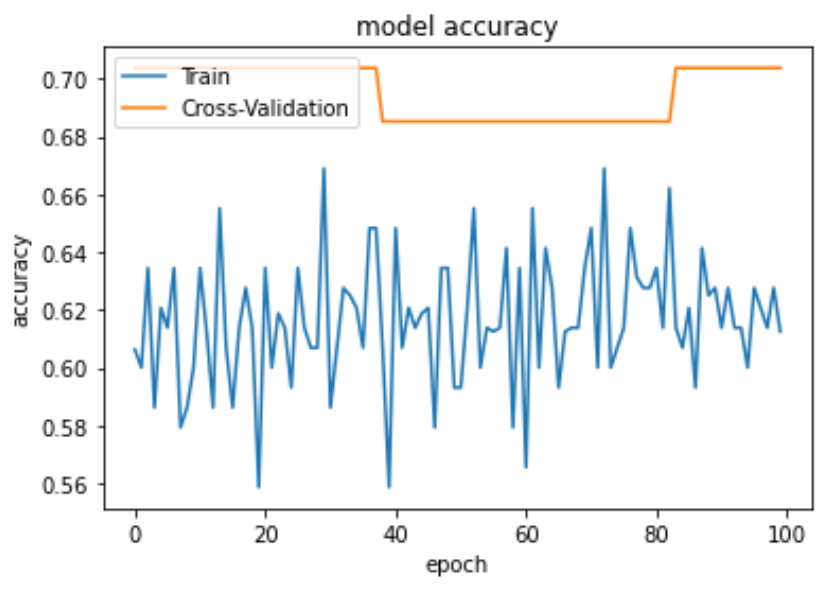

Figure 3 Accuracy for Train data

A confusion matrix (see Fig. 4), also known as error matrix, is a visualization table which that allows to observe the performance of the model. It comprises of true positives, true negatives, false positives, and false negatives which show how well the model is predicting. In this case, the true positive predict by the model are greater compared to the false positives. However, the model could be further improvised by further tunning the hyperparameter in the training data for more elevated results.

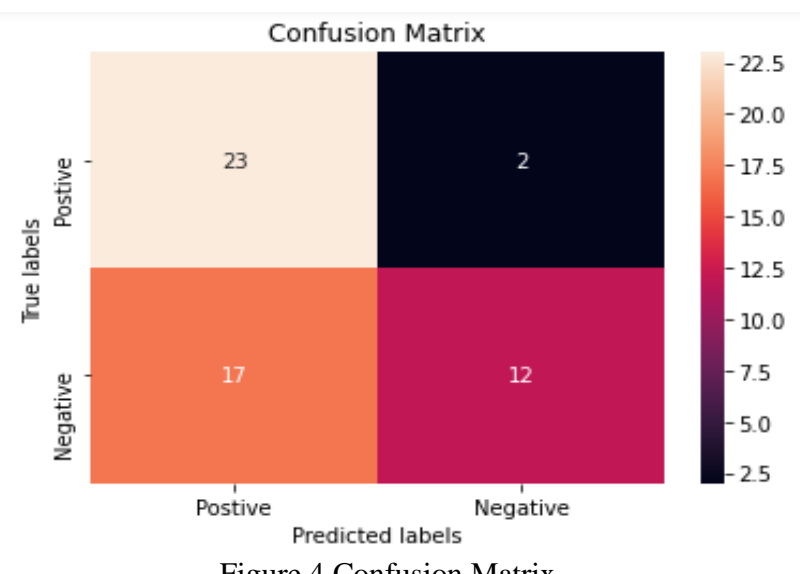

\section{B. Discussion}

In this paper, a recurrent neural network with LSTM was employed for predicting fatigue using two sensors (IMU, HR). We demonstrated that neural networks can be used to predict fatigue. The study provides that fatigue for manual material handling could be employed with a large dataset with multiple sensor fusion. In the LSTM network we used three dense layers and with epoch set to 100 and batch size of 16 to train and fit the NN. However, the results show that there was an increase in the accuracy in the test data. The $\mathrm{NN}$ can be used to predict higher class instead of fatigue vs non-fatigue. The limitations to this work were the small size of the data set which does not represent a convincing sample of the fatigue prediction. In our estimation the research can capitalize in the framework and findings by elevating the size of the data set. 


\section{CONCLUSION}

Fatigue prediction is important in a manufacturing environment to reduce the risk of WMSD and also to prevent from decreased productivity. In this paper, recurrent neural network was employed to predict fatigue from the medium of sensors. However, compared to the literature the results are not very convincing, but one can argue that neural network is data hungry. This dataset was considerably a small dataset.

\section{References}

[1] N. K. Vøllestad, "Measurement of human muscle fatigue.," Journal of neuroscience methods, no. 74(2), pp. 219-227, 1997.

[2] S. Blayney, "Industrial Fatigue and the Productive Body: the Science of Work in Britain," Social History of Medicine, vol. 32, no. 2, p. 310-328, 2017.

[3] M. Sharpe, L. Archard, J. Banatvala, L. Borysiewic, A. Clare, A David and R. Edward, "A Report-Chronic Fatigue Syndrome: Guidelines for Research," Journal of royal society of medicine, vol. 84, no. 2, pp. 118-121., 1991.

[4] M. Looze, , T. Bosch and J. Die€en, "Manifestations of shoulder fatigue in," Ergonomics, vol. 52, no. 4, pp. 428-437, 2009.

[5] M. Yung, "Fatigue at the Workplace: Measurement and Temporal Development. PhD Dissertation," University of Waterloo, 2016. [Online]. https://uwspace.uwaterloo.ca/handle/10012/10119.

[6] K. Saito, "Measurement of fatigue in industries," Industrial Health, vol. 37, no. 2, pp. 134-142, 1999.

[7] P. Gust, U. Müller2 and N. Feller, "Field Study on the Application of a Simulation-Based Software Tool for the Strain-Based Staffing in Industrial Manufacturing," Advances in Applied Digital Human Modeling and Simulation. , vol. 481, pp. 3-12, 2017.

[8] M. Zhang, L. A. Murphy, D. Fang and A. J. Caban-Martinez, "Influence of fatigue on construction workers' physical and cognitive function," Occupational Medicine, vol. 65, no. 3, pp. 245-250, 2015.

[9] M. Loriol, "A sociological stance on fatigue and tiredness: Social inequalities, norms and representations," Neurophysiologie Clinique/Clinical Neurophysiology, vol. 47, no. 2, pp. 87-94, 2017.

[10] E. B., Fatigue: Epidemiology and Social/Industrial Aspects, Tokyo: Springer, Tokyo, 2008, pp. 17-31.

[11] O. Kajimoto, Development of a Method of Evaluation of Fatigue and Its Economic Impacts, Tokyo: Springer, Tokyo, 2008.

[12] Ricci , E. Chee, , A. L. Lorandeau, and J. Berger, "Fatigue in the US workforce: Prevalence and implications," vol. 49, no. 1, pp. 110, Journal of Occupational and Environmental Medicine.

[13] OSHA, "Census of Fatal Occupational Injuries Charts," 2017.

[14] C. Wiktorin, L. Karlqvist and J. Winkel, "Validity of self-reported exposures to work postures and manual materials handling," Scandinavian Journal of Work, Environment and Health, no. 19, pp. 208-2014, 1993.

[15] B. G, "Scaling experiences during work: perceived exertion and difficulty.," Handbook of Human Factors and Ergonomics Methods, pp. 1-7, 2004.

[16] A. E and G. F, "Perceived fatigue during physical work: an experimental evaluation of a fatigue inventory," International Journal of Industrial Ergonomics, no. 21, pp. 117-131, 1998.

[17] C. P, I. Penner, C. Raselli, M. Stöcklin, K. Opwis, Kappos and P. Calabrese, "The Fatigue Scale for Motor and Cognitive Functions (FSMC): validation of a new instrument to assess multiple sclerosis-related fatigue," Multiple Sclerosis Journal, vol. 15, no. 12 , pp. $1509-1517,2009$

[18] G. LI and P. BUCKLE, "Current techniques for assessing physical exposure to work-related musculoskeletal risks, with emphasis on posture-based methods," Ergonomics, vol. 42, no. 5, pp. 674-695, 1999.
[19] L. McAtamney and E. Corlett, "RULA: a survey method for the investigation of work-related upper limb disorders," Applied Ergonomics, vol. 24, no. 2, pp. 91-99, 1993.

[20] Stanton, N, Hedge A, Brookhuis K, Salas E and Hendricks, Handbook of, CRC Press, 2004

[21] S. Rodgers, "Muscle fatigue assessment: functional job analysis technique," in Handbook of Human Factors and Ergonomics Methods, CRC Press, 2005, p. 12.1-12.10.

[22] H. Isa, S. Kamat, A. Rohana, A. Saptari, and M. Shahrizan, "Analysis of muscle activity using surface electromyography for muscle performance in manual lifting task," Applied Mechanics and Materials, vol. 564, pp. 644-649, 2014.

[23] N. Brown, S. Bichler,, M. Fiedler and W. Alt, "Fatigue detection in strength training using three-dimensional accelerometry and principal component analysis," Sports biomechanics, vol. 15, no. 2, pp. 139-150, 2016.

[24] J. Kider , K. Pollock and A. Safonova, "A data-driven appearance model for human fatigue," in ACM SIGGRAPH/Eurographics Symposium on Computer Animation, 2011

[25] J. T, K. J. K. Pollock and A. Safonova, "A Data-Driven Apperance Model for Human Fatigue," in ACM SIGGRAPH Symposium on Computer Animation, Vancouver, 2011.

[26] P. Pramanick and C. Sarkar, "Defatgiue: Online non-intrusive fatigue detection by robot co-worker," in 27th IEEE International Symposium on Robot and Human Interactive Communication (RO-MAN), 2018

[27] M. Ulinskas, R. Damaševičius, , R. Maskeliūnas, and M. Woźniak,, "Recognition of human daytime fatigue using keystroke data," Procedia Computer Science, vol. 130, pp. 947-952, 2018.

[28] Z. S. Maman, Y.-J. Chen, A. Baghdadi, S. Lombardo, L. Cavuoto and F. Megahed, "A data analytic framework for physical fatigue management using wearable sensors," Expert Systems With Applications, vol. 155, 2020

[29] G. Petneházi, "Recurrent neural networks for time series forecasting," 2019.

[30] s. Hochreiter and J. Schmidhuber,, "Long Short-Term Memory," Neural Computation,," vol. 9, no. 8, pp. 1735-1780, 1997.

[31] M. Abadi, P. Barham, J. Chen, D. Chen, A. Davis, J. Dean, M. Devin and S. Ghemawat, "TensorFlow:A System for Large-Scale Machine Learning.," 2016.

[32] S. a. H. A. a. R. f. P. (SHARP), "Work-related musculoskeletal disorders of the neck, back, and upper extremity in Washington State," Washington State Department of Labor and, 2005. 